\title{
Infimal generators and monotone sublinear operators
}

\author{
SEMËN S. KUTATELADZE*
}

ABSTRACT. We provide a version of Korovkin-type theorems for monotone sublinear operators in vector lattices and discuss the possibilities of further extensions and generalizations.

Keywords: Supremal generator, Korovkin-type theorem, sublinear operator.

2020 Mathematics Subject Classification: 41A36, 46N99.

Dedicated to Professor Francesco Altomare, on occasion of his 70th birthday, with esteem and friendship.

\section{INTRODUCTION}

S. G. Gal and C. P. Niculescu recently proved a Korovkin-type theorem for monotone sublinear operators in spaces of continuous functions in $N$-dimensions [1, Theorem 2]. As the test functions they use the coordinate projections and the sum of their squares whose span is a classical supremal generator in $C(Q)$ with $Q$ a compact subset of the $N$-dimensional Euclidean space $\mathbb{R}^{N}$. In this short note, we extend this theorem to order convergence.

\section{The MAIn Result}

We will proceed with a vector sublattice $X$ of a Dedekind complete vector lattice $Y$. $\operatorname{Re}-$ call that a subset $H$ of $X$ is a supremal generator of $X_{+}$with respect to $Y$ provided that $x=\sup _{Y}\{h \in H: h \leq x\}$ for all $x \in X_{+}$, where as usual $X_{+}=\{x \in X: x \geq 0\}$. Using the reverse order, we define an infimal generator of $X_{+}$with respect to $Y$ for convenience. We let $L_{+}(X, Y)$ stand for the set of positive linear operators from $X$ to $Y$. Assume that $\operatorname{Id}_{Y}$ is the identity operator on $Y$. Then, the order interval $\left[0, \mathrm{Id}_{Y}\right]$ is the set of multipliers of $Y$.

Theorem 2.1. Let $H$ be a convex cone in $X$. Then, the following are equivalent:

(1) $H$ is an infimal generator of $X_{+}$with respect to $Y$;

(2) there is a multiplier $\lambda \in\left[0, \mathrm{Id}_{Y}\right]$ such that $o-\lim \sup _{\alpha \in \mathrm{A}} P_{\alpha}(x)=\lambda x$ for all $x \in X_{+}$and every net $\left(P_{\alpha}\right)_{\alpha \in \mathrm{A}}$ of monotone sublinear operators from $X$ to $Y$ such that $o-\lim \sup _{\alpha} P_{\alpha \in \mathrm{A}}(h) \leq h$ for all $h \in H$;

(3) $H$ is cofinal and if $T \in L_{+}(X, Y)$ such that $T h \leq h$ for all $h \in H$; then $T$ is a multiplier in $Y$. Proof. (1) $\rightarrow$ (2): Clearly, $P(x):=o-\lim \sup _{\alpha \in \mathrm{A}} P_{\alpha}(x)$ exists for every $x \in X$. So, $P: X \rightarrow$ $Y$ is a monotone sublinear operator and $P(x)=\sup \{T x: T \in \partial(P)\}$, where $\partial(P)$ is the subdifferential of $P$.

Take $T \in \partial(P), x \in X_{+}$, and $h \geq x, h \in H$. Since $H$ is an infimal generator of $X_{+}$with respect to $Y$ and $T h \leq P(h) \leq h$; therefore, $T x \leq x$ by the operator principle of preservation of

Received: 15.11.2020; Accepted: 18.01.2021; Published Online: 01.03.2021

*Corresponding author: Semën S. Kutateladze; sskut@math.nsc.ru

DOI: $10.33205 / \mathrm{cma} .826134$ 
inequalities [2, Theorem 2.1]. Consequently, $T \in\left[0, \operatorname{Id}_{Y}\right]$ and so $P(x)=\lambda x$ for all $x \in X_{+}$since the pointwise supremum of multipliers is a multiplier itself.

(2) $\rightarrow$ (3): This is obvious.

(3) $\rightarrow(1)$ : Let $q_{H}(x):=\inf \{h \in H: h \geq x\}$. Then, $q_{H}$ is a monotone sublinear operator on $X$ and $\partial\left(q_{H}\right)=\left\{T \in L_{+}(X, Y):(\forall h \in H) T h \leq h\right\}$. By (3), $q_{H}$ is a multiplier itself.

\section{COMMENTS AND EXTENSIONS}

In this section, we will discuss applications and extensions of Theorem 2.1 using the terminology and techniques of vector lattices, subdifferential calculus, and Boolean-valued analysis as presented in [3] and [4].

The ideas behind the main theorem make it possible to abstract the effects of monotone sublinear approximations along all lines thoroughly presented in [5]. For instance, if $H$ is a finite-dimensional subspace of $X$, then $X$ is a vector lattice of bounded elements. Moreover, $H$ is coinitial to $X$ (in fact, a supremal generator as well) and we have an analog of Theorem 2.1 for relatively uniform convergence to the embedding of $X$ to $Y$. This yields uniform approximation by monotone sublinear operators in spaces of bounded continuous functions on compact subsets of finite-dimensional Euclidean space.

Many opportunities are open by Boolean valued analysis for Korovkin-type results in the realm of ordered modules over the ring of orthomorphisms of a Dedekind complete vector lattice $Y$. We can consider approximation of the embedding by monotone module-sublinear operators along the lines of Theorem 2.1. The rest of the matter is that the modular situation can be lifted to the Boolean valued universe $\mathbb{V}^{\mathbb{B}}$ over the base $\mathbb{B}$ of $Y$ (i.e., the set of band projections in $Y$ ). Since by transfer Theorem 2.1 and its analogs are valid inside $\mathbb{V}^{\mathbb{B}}$, we can use their Boolean valued interpretations which yield, for instance, some Korovkin-type theorems for approximation of extensional continuous vector-valued functions on the so-called procompact spaces which are usually noncompact (for instance, the order intervals between two Lebesgue measurable functions).

We will not dwell on all these new model-theoretic opportunities since the possibilities of their application for the needs of the working mathematician seem dim these days.

\section{REFERENCES}

[1] S. G. Gal, C. P. Niculescu: A nonlinear extension of Korovkin's theorem, Mediterranean Journal of Mathematics, 17 (5) (2020), Art. no. 145, 14 pages.

[2] S. Kutateladze, A. Rubinov: Minkowski duality and its applications, Russian Math. Surveys, 27 (3) (1972), no. 3, 137-191.

[3] A. Kusraev, S. Kutateladze: Subdifferentials: Theory and Applications, Kluwer, Dordrecht (1995).

[4] A. Kusraev, S. Kutateladze: Boolean Valued Analysis, Kluwer, Dordrecht (1999).

[5] F. Altomare, M. Campiti: Korovkin-type Approximation Theory and Its Applications, De Gruyter, Berlin and New York (1994).

SEMËN S. KutATELADZE

SOBOLEV INSTITUTE OF MATHEMATICS

KOPtyUg AVENUE No 4, 630090, NOVOSIBIRSK, RUSSIA

ORCID: 0000-0002-5306-2788

E-mail address: sskut@math.nsc.ru 